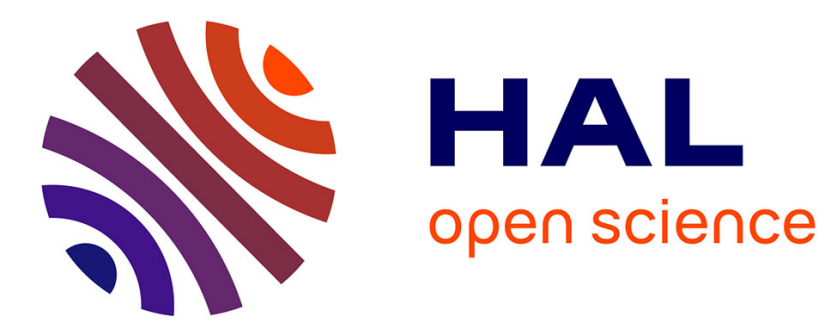

\title{
Broadening low-frequency bandgaps in locally resonant piezoelectric metamaterials by negative capacitance
}

\author{
Kaijun Yi, Manuel Collet
}

\section{To cite this version:}

Kaijun Yi, Manuel Collet. Broadening low-frequency bandgaps in locally resonant piezoelectric metamaterials by negative capacitance. Journal of Sound and Vibration, In press, 10.1016/j.jsv.2020.115837 . hal-03026053

\section{HAL Id: hal-03026053 \\ https://hal.science/hal-03026053}

Submitted on 26 Nov 2020

HAL is a multi-disciplinary open access archive for the deposit and dissemination of scientific research documents, whether they are published or not. The documents may come from teaching and research institutions in France or abroad, or from public or private research centers.
L'archive ouverte pluridisciplinaire HAL, est destinée au dépôt et à la diffusion de documents scientifiques de niveau recherche, publiés ou non, émanant des établissements d'enseignement et de recherche français ou étrangers, des laboratoires publics ou privés. 


\title{
Broadening low-frequency bandgaps in locally resonant piezoelectric metamaterials by negative capacitance
}

\author{
Kaijun $\mathrm{Yi}^{\mathrm{a}, *}$, Manuel Collet ${ }^{\mathrm{b}}$ \\ ${ }^{a}$ School of Aerospace Engineering, Beijing Institute of Technology, Beijing 100081, PR China \\ ${ }^{b}$ Univ Lyon, Ecole Centrale de Lyon, ENISE, ENTPE, CNRS, Laboratoire de Tribologie et Dynamique \\ des Systèmes LTDS UMR5513, F-69134 Ecully, France
}

\begin{abstract}
This paper combines negative capacitance (NC) with inductance (L) to enlarge lowfrequency bandgap width in locally resonant piezoelectric metamaterials. The studied metamaterials are obtained by directly bonding patches on the surfaces of host structures, then connecting patches to shunts. Shunts with NC and L in series and in parallel are both studied. Analytical expressions of the bandgap ranges are derived, which reveal that the bandgap size is increased not simply because the NC enhancing the material's electro-mechanical coupling factor, but in a more complicated way. Parametric studies are performed to analytically investigate the tuning properties of the LR bandgap by NC. Results demonstrate that by modifying NC value, the LR bandgap size can be significantly increased. Numerical simulations are done to verify the effects of the broadened bandgap on vibration transmission and reveal the limitations of the used analytical model. Practical implementation of the shunts are also discussed, recommendations on choosing the shunt configurations and $\mathrm{NC}$ values are given. This paper gives a theoretical guideline on designing piezoelectric metamaterials with bandgap effects at desired frequency ranges for practical applications like low-frequency vibration and noise reduction or isolation. Keywords: metamaterial, bandgap, piezoelectric material, negative capacitance, vibration and noise control
\end{abstract}

\footnotetext{
*Corresponding author

Email address: kaijun.yi@bit.edu.cn (Kaijun Yi)
} 


\section{Introduction}

In recent years, the emerging metamaterials based on local resonators provide new ways to deal with low-frequency vibration and noise issues [1-6]. Metamaterials present sub-wavelength bandgaps (at frequencies much lower than the first Bragg bandgap), within which wave propagation is prohibited. Therefore, using the bandgaps, one can totally remove the resonant modes or isolate vibration transmission. Metamaterials based on passive resonators are first studied. However, they have narrow bandgaps and are not adaptable to working condition or environment changes. These drawbacks limit they applications in practice. Recently, piezoelectric materials with external shunting circuits have been integrated into structures, results in so-called piezoelectric (smart) metamaterials [7-12]. In such metamaterials, the locations and widths of bandgaps can be easily tuned by just varying the circuits, no modification is needed on the mechanical structures. Different shunting circuits, such as resonant shunts and negative capacitance (NC) circuits, have been proposed to create and tune bandgaps in piezoelectric metamaterials. However, there are still challenges in creating wide and controllable bandgaps at low frequencies in these metamaterials, because of the necessity of using large inductance or lack of robustness. In this paper, we are going to demonstrate that combining resonant shunts with NC could be an effective way to realize wide and tunable bandgaps at low frequencies.

Piezoelectric shunt techniques are first proposed for vibration reduction of flexible structures. Hagood and von Flotow [13] demonstrated that a piezoelectric patch connected with an inductance $(\mathrm{L})$ behaves like a mechanical resonator. This is because the $\mathrm{L}$ and the intrinsic capacitance of the patch form a resonant shunt. Based on this feature, patches shunted with L are periodically bonded on a bar to create locally resonant (LR) bandgaps in Thorp et al's work [14]. It was shown that bandgaps are created in the bar near the resonance frequency of the circuit at sub-wavelength frequencies. The location of the bandgap can be tuned by varying the L value. Airoldi and Ruzzene [15] 
studied the effective bending stiffness of a periodic beam with distributed patches shunted by $\mathrm{L}$ and resistance $(\mathrm{R})$. They demonstrated that the beam's effective bending stiffness presents resonant feature near the resonance frequency of the circuit, just like the effective mass of a metamaterial with mechanical resonators does near the resonance frequency. Therefore, it was concluded that the periodic beam with patches shunted by $\mathrm{L}$ is an electro-mechanical version of the locally resonant metamaterials. Following these works, the conception of locally resonant piezoelectric metamaterial is expanded to plates [1620]. However, due to the resonance nature, bandgaps in these metamaterials are narrow. To enlarge LR bandgaps in piezoelectric metamaterials, Wang et al. [21, 22] proposed an amplifier-resonator feedback circuit to broad bandgaps. Numerical and experimental results in their works demonstrate that the bandgap is broadened, but is still not wide enough. The conception of varying shunts' parameters gradually in space to create "rainbow traps" is explored in [23, 24]. This kind of design shows good effects in increasing bandgap ranges, but the number of unit cells needed to realize a "rainbow trap" with acceptable wave isolation level could be large. Shunts with multiple resonance frequencies are also proposed. The multiple resonant effects could be realized using multi-branch shunts [25] or digital circuits with properly designed control law [26, 27]. Limited by the complexity of multi-branch shunts and processing speed in digital circuits, the number of resonance frequency is within 3 at present. Except resonant shunts, NC circuits are also proposed to control bandgaps in piezoelectric metamaterials [28-30]. NC can cancel a part of the intrinsic capacitance of a patch, therefore to enhance the electro-mechanical coupling effect $[31,32]$. Recently, it is also shown that NC can increase or decrease the effective static stiffness of the structure covered by the patches [33]. This property has been explored to enlarge bandgaps in piezoelectric metamaterials. In these applications, patches shunted with NC can be directly bonded on the surfaces of structures [34], the NCs are used to change the local stiffness therefore to tune the bandgaps induced by Bragg scattering effects. In such a manner, the integrity of the structures is preserved. However, 
in order to obtain wide and low-frequency bandgaps, the NC needs to significantly reduce the stiffness of the host structures, i.e., the NC need to work at the vicinity of its unstable zone, which is impractical. In other studies [35-38], patches shunted with NC are used to control the stiffness of local resonators in passive metamaterials, therefore to broaden the bandgaps caused by passive resonators. In this way, it is more easy to obtain wide bandgaps at low frequencies, however extra local resonators need to be added into the system, increasing the total mass and volume or break integrity of the host structure, which are strictly limited in many practical applications.

In this paper, $\mathrm{L}$ and $\mathrm{NC}$ are combined to realize low and wide bandgaps in piezoelectric metamaterials. It should be noted that combining $\mathrm{L}$ and $\mathrm{NC}$ are previously studied for vibration reduction of structural modes [39, 40], but few works are done to increase bandgap size. The studied metamaterials are obtained by directly bonding patches on the surfaces of a host structure, therefore to avoid adding too much extra mass and keep the integrity of the original structure. A beam is used as the host structure in this study. Note that the proposed conception in this paper can be naturally extended to other types of structures, like plates and shells. Combining NC with $\mathrm{L}$ in the shunts remains the resonant nature of the shunts. Therefore, LR bandgaps at low frequencies can still be obtained in the proposed piezoelectric metamaterials. Effects of NC on the LR bandgap are analytically and numerically studied in the following sections.

\section{Theoretical analysis}

\subsection{Shunting configurations}

NC could be connected with $\mathrm{L}$ in series or in parallel, as shown in Fig. 1. In these figures, $C_{0}$ indicates NC, the piezoelectric patch made of PZT is represented by a current source $I_{e q}(t)$ in parallel with a capacitance $C_{p}^{s}$. All these two shunting configurations and the case with only $\mathrm{L}$ are studied in this paper. The impedance $\left(Z_{s u}\right)$ of these different shunts are summarized in Table 1 . In the table, $\mathrm{j}$ is the unit of complex number and $\omega$ is 
the angular frequency.

(a)

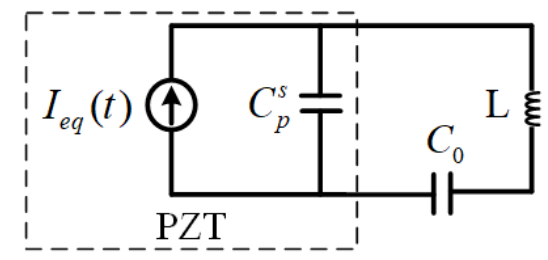

(b)

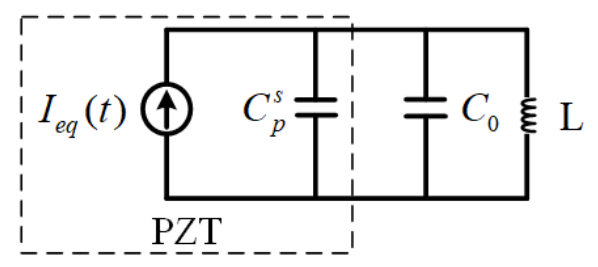

Figure 1: Different shunting configurations: (a) L in series with NC; (b) L in parallel with NC. $C_{0}$ indicates NC. The piezoelectric patch made of PZT is represented by a current source $I_{e q}(t)$ in parallel with a capacitance $C_{p}^{s}$.

Table 1: Impedance of different shunts

\begin{tabular}{lll}
\hline only L & $\begin{array}{l}\mathrm{L} \text { and NC } \\
\text { in series }\end{array}$ & $\begin{array}{l}\mathrm{L} \text { and NC } \\
\text { in parallel }\end{array}$ \\
\hline$Z_{s u}=\mathrm{j} \omega L$ & $\frac{-\omega^{2} L C_{0}+1}{\mathrm{j} \omega C_{0}}$ & $\frac{\mathrm{j} \omega L}{\left(1-\omega^{2} L C_{0}\right)}$ \\
\hline
\end{tabular}

\subsection{Governing equations}

Figure 2 (a) shows the studied metamaterial beam with periodically distributed shunted piezoelectric patches, a unit cell of it is illustrated in Fig. 2 (b).

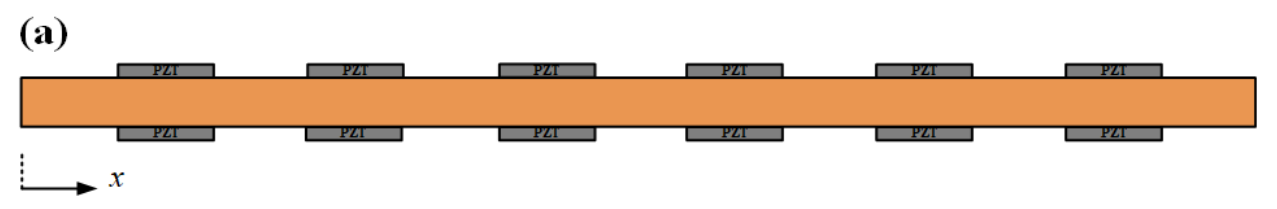

(b)

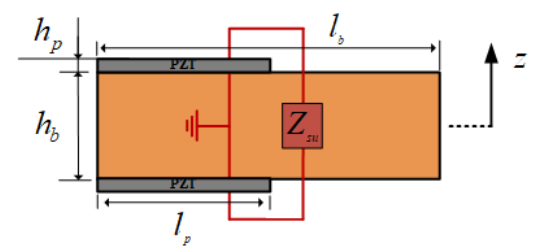

Figure 2: (a) The studied piezoelectric metamaterial beam, (b) a unit cell of the metamaterial.

We consider the flexural waves in the metamaterial beam. The beam is slender, EulerBernoulli beam theory could be used. Under harmonic excitation, the equations of the transverse motion of the beam $w(x)$ can be written as: 


$$
\frac{\partial^{2}}{\partial x^{2}}\left[D(x) \frac{\partial^{2} w(x)}{\partial x^{2}}\right]-\omega^{2} m(x) w(x)=0
$$

where, $D(x)$ is the bending stiffness of the beam, $m(x)$ is the mass per unit area. The unit cell has a piece-wise configuration, the length of the period is $l_{b}$, as shown in Fig. 2(b). The expressions for the piece-wise bending stiffness in a unit cell are:

$$
D(x)=\left\{\begin{array}{l}
D_{1}=D_{b}+\frac{E_{p}(\omega) b\left[\left(h_{b}+2 h_{p}\right)^{3}-h_{b}^{3}\right]}{12}, 0 \leq x<l_{p} \\
D_{2}=D_{b}, l_{p} \leq x<l_{b}
\end{array}\right.
$$

in which, $D_{b}=E_{b} b h_{b}^{3} / 12$ is the bending stiffness of the host beam, $E_{b}$ is the Young's modulus of the host beam, $E_{p}$ is the effective Young's modulus of the shunted piezoelectric patch [13], expression of it is:

$$
E_{p}(\omega)=E_{p}^{o c}\left(1-\frac{k_{31}^{2}}{1+\mathrm{j} \omega C_{p}^{s} Z_{s u}(\omega)}\right),
$$

in which, $k_{31}=d_{31} \sqrt{E_{p}^{s c} / \varepsilon_{3}^{\sigma}}$ is the extensional coupling factor of the patch [41], $E_{p}^{o c}=$ $E_{p}^{s c} /\left(1-k_{31}^{2}\right)$ is the patch's Youngs' modulus under open-circuited (OC) condition, $C_{p}^{s}=$ $C_{p}^{T}\left(1-k_{31}^{2}\right)$ is the intrinsic capacitance of the patch under constant strain and $C_{p}^{T}=$ $A_{p} \varepsilon_{3}^{\sigma} / h_{p}$, with $A_{p}$ the area of the electrode.

The linear mass is:

$$
m(x)=\left\{\begin{array}{l}
m_{1}=\rho_{b} b h_{b}+2 \rho_{p} b h_{p}, 0 \leq x<l_{p} \\
m_{2}=\rho_{b} b h_{b}, \quad l_{p} \leq x<l_{b}
\end{array}\right.
$$

in which, $\rho_{b}$ and $\rho_{p}$ are the densities of the host beam and the patch, respectively. 


\subsection{Transfer matrix method}

The transfer matrix (TM) method is used to calculate the dispersion curves. The sate vector $\boldsymbol{y}(\boldsymbol{x})$ is used in the TM method. Therefore, Eq. (1) is rewritten into the form:

$$
\frac{\partial \boldsymbol{y}(\boldsymbol{x})}{\partial x}=\mathbf{C}(x, \omega) \boldsymbol{y}(\boldsymbol{x})
$$

where, $\mathbf{C}(x, \omega)$ is a periodic and piecewise function:

$$
\mathbf{C}(x, \omega)=\left\{\begin{array}{l}
\mathbf{C}_{1}(x, \omega), 0 \leq x<l_{p} \\
\mathbf{C}_{2}(x, \omega), l_{p} \leq x<l_{b}
\end{array}\right.
$$

For the transverse motion, the state vector is $\boldsymbol{y}(\boldsymbol{x})=\left[\begin{array}{lll}w(x) & \theta(x) & M(x)\end{array}(x)\right]^{T}$, in which, the slope is $\theta(x)=\partial w(x) / \partial x$, the bending moment is $M(x)=-D(x) \partial^{2} w(x) / \partial x^{2}$ and the shear force is $Q(x)=-D(x) \partial^{3} w(x) / \partial x^{3}$, and:

$$
\mathbf{C}_{i}(x, \omega)=\left[\begin{array}{cccc}
0 & 1 & 0 & 0 \\
0 & 0 & -\frac{1}{D_{i}(x)} & 0 \\
0 & 0 & 0 & 1 \\
-\omega^{2} m_{i}(x) & 0 & 0 & 0
\end{array}\right], i=1,2
$$

Equation (3) describes a 1D medium with periodic coefficients. Using the Floquet theorem, the state vectors of the two ends of a unit cell are related through

$$
y\left(x+l_{b}\right)=\lambda y(x)
$$

in which, $\lambda=e^{\mathrm{j} k l_{b}}$ is the Floquet multiplier, with $k$ the wavenumber. 
The state vectors of the two ends can also be related through a TM T:

$$
y\left(x+l_{b}\right)=\mathrm{T} y(x)
$$

Eqs. (5) and (6) together form an eigenvalue problem for the Floquet multiplier and the state vector:

$$
(\mathbf{T}-\lambda \mathbf{I}) \boldsymbol{y}(\boldsymbol{x})=0
$$

in which, $\mathbf{I}$ is a diagonal matrix with all terms equal to one.

For the TM between the two ends of the studied unite cell (Fig. 2(b)), considering the continuities of displacement and force at the interface between the two segments, $\mathbf{T}$ is given by:

$$
\mathbf{T}=\mathbf{T}_{2} \mathbf{T}_{1}
$$

where, $\mathbf{T}_{1}$ relates the two state vectors at $x=0$ and $x=l_{p}$, while $\mathbf{T}_{2}$ relates the two state vectors at $x=l_{p}$ and $x=l_{b}$. $\mathbf{T}_{1}$ and $\mathbf{T}_{2}$ can be expressed as [15]:

$$
\mathbf{T}_{1}=e^{l_{b} \mathbf{C}_{1}}, \mathbf{T}_{2}=e^{\left(l_{b}-l_{p}\right) \mathbf{C}_{2}}
$$

After obtaining the TM, the Floquet multiplier can be obtained by solving the eigenvalue problem in Eq. (7) when the frequency is given, the wavenumber is then deduced according to $\lambda=e^{\mathrm{j} k l_{b}}$.

\subsection{Analytical estimation of LR bandgap range}

The bandgaps in passive locally resonant metamaterials are caused by negative parameters like negative density or bulk modulus [42]. In locally resonant piezoelectric metamaterials, it has been mentioned that bandgaps for flexural waves are associated with negative bending stiffness [43]. However, no analytical expressions have yet been 
given to directly estimate the ranges of bandgaps. Such expressions could be very useful to analyze the influences of $\mathrm{NC}$ and other parameters on the bandgap sizes and locations.

Using the effective medium theory, the effective bending stiffness of the whole unit cell can be approximately expressed as [15]:

$$
D_{e f f}(\omega)=\frac{D_{A} D_{b}}{(1-\chi) D_{A}+\chi D_{b}}
$$

in which, $\chi=l_{p} / l_{b}$, it is the covering ratio of the patch, $D_{A}$ is the bending stiffness of the part covered by patches, the expression of it is:

$$
D_{A}(\omega)=D_{b}+\frac{D_{p}^{s c}}{1-k_{31}^{2}}-\frac{k_{31}^{2}}{1-k_{31}^{2}} D_{p}^{s c} \frac{1}{1+\mathrm{j} \omega Z_{s u} C_{p}^{s}}
$$

in which, $D_{p}^{s c}=E_{p}^{s c} b\left[\left(h_{b}+2 h_{p}\right)^{3}-h_{b}^{3}\right] / 12$ is the bending moment of the patches under SC condition.

When the configuration of the shunt is given, namely, the expression of $Z_{s u}$ is determined, according to Eqs. (10) and (11), one can easily deduce the expressions of the effective bending stiffness and the range where the stiffness is negative. To make the analysis more general, the following non-dimensional parameters are used hereinafter along with the previously defined $\chi=l_{p} / l_{b}$ :

$$
\gamma=D_{b} / D_{p}^{s c}, \alpha=C_{0} / C_{p}^{s}
$$

$\alpha$ is a real number, it could be regarded as the non-dimensional $\mathrm{NC}$ value.

For the case with only $\mathrm{L}$ in the shunt, the frequency range where the effective bending stiffness becomes negative is

$$
\omega_{L C} \sqrt{1-\frac{k_{31}^{2}}{\gamma\left(1-k_{31}^{2}\right)+1}}<\omega<\omega_{L C} \sqrt{\frac{(\gamma+1-\chi)\left(1-k_{31}^{2}\right)}{\gamma\left(1-k_{31}^{2}\right)+1-\chi}}
$$


in which, $\omega_{L C}=1 / \sqrt{L C_{p}^{s}}$ is the resonance frequency of the circuit. Eq. (12) also gives the bandgap range (which will be verified later in this section), it can be seen that the lower boundary of the bandgap depends on the resonance frequency of the circuit $\left(\omega_{L C}\right)$, the ratio of the bending stiffness of the host beam to that of the short-circuited patch $(\gamma)$ and the extensional coupling factor $\left(k_{31}\right)$. The upper boundary not only depends on the three factors listed above, but also depends on the covering ratio of the patch $(\chi)$. If $\chi=1$, which means the patches cover all the surfaces of the host beam, the upper boundary is exactly equal to $\omega_{L C}$, this is the case studied in [44]. If $\chi<1$, namely, the patches are shorter than the host beam in a unit cell, the upper boundary is lower than $\omega_{L C}$

Due to the active nature of NCs [29], they may cause stability issues in some circumstances, which must be avoided in applications. Therefore, before presenting the expressions of bandgap ranges for the cases with NCs in the shunts, the stable zone for the $\mathrm{NC}$ values are shown first. In this paper, the $\mathrm{L}$ and $\mathrm{R}$ values are not limiter to be positive, since one can obtain negative L and $\mathrm{R}$ using synthetic or digital circuits. 'Therefore, the studied piezoelectric metamaterial beam is stable as long as a positive bending stiffnes $:$ it is guaranteed when $\omega=0$. According to this, we can deduce the stable zone for NC. It is found that the stable zone of NC is the same no matter it is in series or in parallel with L. The stable zone is

$$
\alpha<-\frac{\gamma\left(1-k_{31}^{2}\right)+1}{(\gamma+1)\left(1-k_{31}^{2}\right)}, \text { or } \alpha>-1
$$

The stable zone includes two sets, as shown graphically in Fig. 3. The limit of the left set depends on $\gamma, k_{31}$ and $C_{p}^{s}$ (recall that $\alpha=C_{0} / C_{p}^{s}$ ), the limit of the right set only depends on $C_{p}^{s}$.

When the shunts is composed of $\mathrm{L}$ in series with $\mathrm{NC}$, the equivalent capacitance 


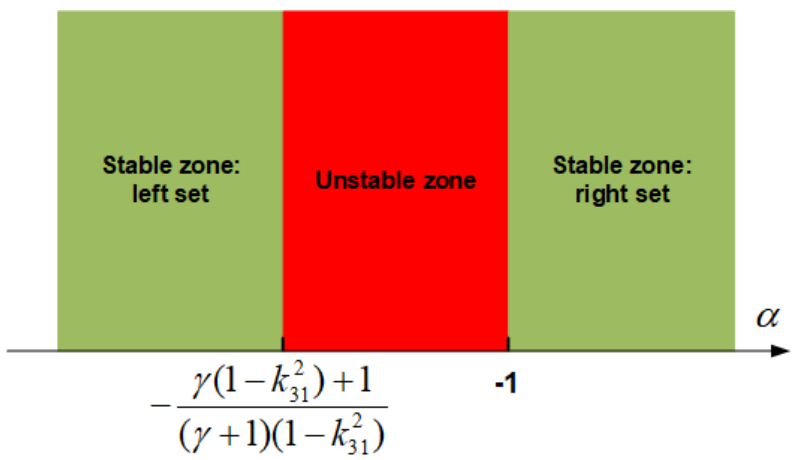

Figure 3: Graphical illustration of the stable zone in Eq. (13).

of the circuit becomes $C_{e q}=\alpha C_{p}^{s} /(1+\alpha)$ (see Fig. 1 (a)). Consequently, the resonance frequency of the circuit is $\omega_{L C}=1 / \sqrt{L C_{e q}}$. If $\mathrm{L}$ is fixed, resonance frequency of the circuit will change with the $\mathrm{NC}$ value. Usually, our interest is to obtain bandgaps at targeted frequency ranges. Accordingly, in our studies, we assume that resonance frequency of the circuit is fixed, which means $\mathrm{L}$ is varying according to the applied $\mathrm{NC}$ value. In this case, the bandgap range becomes

$$
\left\{\begin{array}{l}
\omega_{L C} \sqrt{1-\frac{1}{1+\alpha} \frac{k_{31}^{2}}{\gamma\left(1-k_{31}^{2}\right)+1}}<\omega<\omega_{L C} \sqrt{\frac{\gamma\left(1-k_{31}^{2}\right)+(1-\chi)\left(1-\frac{k_{31}^{2}}{1+\alpha}\right)}{\gamma\left(1-k_{31}^{2}\right)+1-\chi}}, \alpha>-1 \\
\omega_{L C} \sqrt{\frac{\gamma\left(1-k_{31}^{2}\right)+(1-\chi)\left(1-\frac{k_{31}^{2}}{1+\alpha}\right)}{\gamma\left(1-k_{31}^{2}\right)+1-\chi}}<\omega<\omega_{L C} \sqrt{1-\frac{1}{1+\alpha} \frac{k_{31}^{2}}{\gamma\left(1-k_{31}^{2}\right)+1}}, \alpha<-\frac{\gamma\left(1-k_{31}^{2}\right)+1}{(\gamma+1)\left(1-k_{31}^{2}\right)}
\end{array}\right.
$$

From the above expressions, it can be seem that NC influences simultaneously the lower and upper boundaries of the bandgap when $\chi<1$. For the critical situation with $\chi=1$, only the lower or upper boundary (depends on the $\alpha$ value) will be modified by NC. Comparing Eqs. (12) and (14), it is also observed that the effects of $\mathrm{NC}$ on the bandgap boundaries are not simply due to the modification of the material eletro-mechanical coupling factor (which becomes $k_{31} \sqrt{\alpha /(\alpha+1)}$ when the $\mathrm{NC}$ is connected in series).

When $\mathrm{L}$ is in parallel with $\mathrm{NC}$, the equivalent capacitance of the circuit becomes $C_{e q}=\left(1+\alpha_{p}\right) C_{p}^{s}$ (see Fig. 1 (b)). In this case, the bandgap range is 


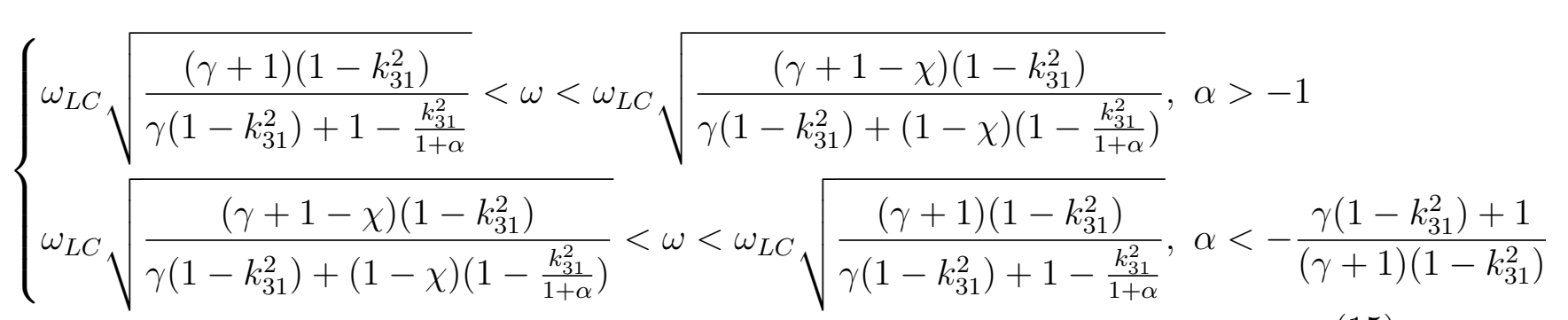

It is also observed that $\mathrm{NC}$ modifies simultaneously the upper and lower boundaries if $\chi<1$, and only one of the boundaries if $\chi=1$.

To verify the accuracy of Eqs. (12), (14) and (15), the negative bending stiffness ranges predicted by them are compared with the bandgap ranges obtained using dispersion curves, the results are shown in Figures 4. In these simulations, the host beam is made of aluminum and the patches are made of PZT-5H, the geometric and material parameters are described in Table 2. For the $\mathrm{NC}$ in series with $\mathrm{L}, \alpha=-1.5$ is used as an example, and for the NC in parallel with $\mathrm{L}, \alpha=-0.7$ is used. The shadowed area in each figure indicates the range where the effective bending stiffness is negative, it is obtained using one of the expressions in Eqs. (12), (14) and (15). It can be observed that the bandgaps completely overlap the negative bending stiffness ranges, which verifies the accuracy of using Eqs. (12), (14) and (15) to predict the bandgaps. It is also observed that the NC enlarges the negative bending stiffness ranges, therefore leading to wider bandgaps. More details on how $\mathrm{NC}$ and other parameters of the piezoelectric metamaterial influence the bandgap will be discussed later.

\subsection{Tuning characteristics of LR bandgap by $N C$}

In this section, first, the influences of $\mathrm{NC}$ value on bandgap ranges are studied. Then, effects of $\mathrm{NC}$ on the wave attenuation ability within bandgaps are analyzed. The bandgap ranges are predicted using Eqs. (14) and (15), the wave attenuation properties within bandgaps are obtained using the TM method.

In the simulations to study the tuning characteristics of bandgap ranges, $\gamma=0.23$ 

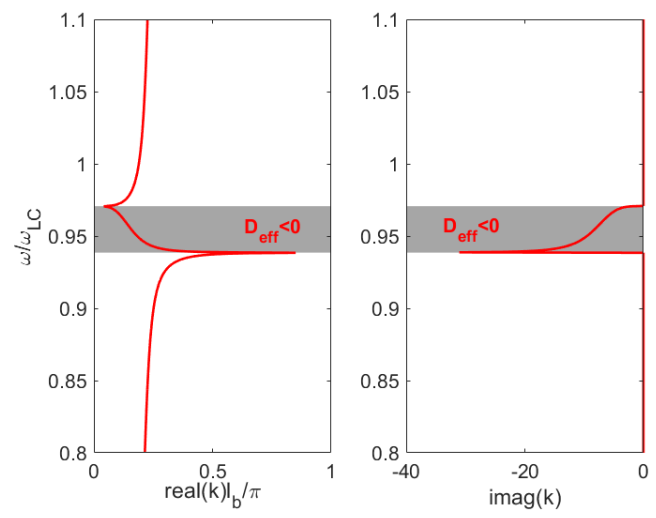

(a)
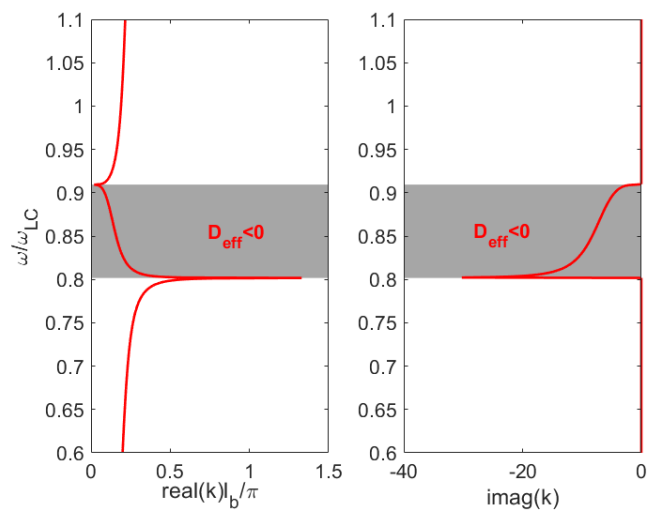

(b)
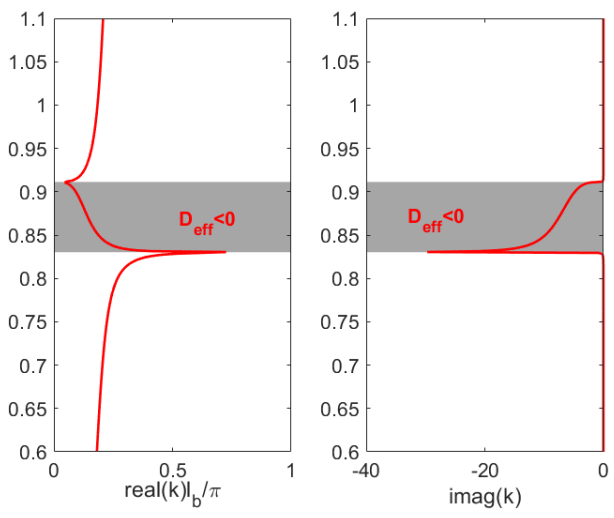

(c)

Figure 4: Dispersion curves of the flexural waves in the piezoelectric metamaterial beam for three different shunts: (a) shunt with only L, (b) shunt with $\mathrm{L}$ and $\mathrm{NC}$ in series, $\alpha=-1.5$ and (c) shunt with $\mathrm{L}$ and $\mathrm{NC}$ in parallel, $\alpha=-0.7$. Shadowed area in each figure corresponding to the frequency range where the negative effective bending stiffness is negative, predicted using one of the expressions in Eqs. (12), (14) and (15). 
Table 2: Geometric and material parameters of the unit cell

\begin{tabular}{lll}
\hline & Beam & Piezoelectric patch \\
\hline Length & $l_{b}=60 \mathrm{~mm}$ & $l_{p}=50 \mathrm{~mm}$ \\
Width & $\mathrm{b}=50 \mathrm{~mm}$ & $\mathrm{~b}=50 \mathrm{~mm}$ \\
Thickness & $h_{b}=3 \mathrm{~mm}$ & $h_{p}=0.5 \mathrm{~mm}$ \\
Young's modulus & $E_{b}=70 \mathrm{Gpa}$ & $E_{p}^{s c}=60.6 \mathrm{Gpa}$ \\
Density & $\rho_{b}=2700 \mathrm{~kg} / \mathrm{m}^{3}$ & $\rho_{p}=7500 \mathrm{~kg} / \mathrm{m}^{3}$ \\
Coupling constant & $\backslash$ & $d_{31}=-2.74 \mathrm{e}-10 \mathrm{C} / \mathrm{N}$ \\
Relative permittivity & $\backslash$ & $\varepsilon_{3}^{\sigma}=3400$ \\
under constant stress & & \\
\hline
\end{tabular}

and $k_{31}=-0.39$ are used, they are corresponding to the dimensional parameters listed in Table 2. For the parameter $\chi$, three different values of it are used, they are $\chi=0.5$, $\chi=0.7$ and $\chi=1$, the aim of using 3 different $\chi$ values is to reveal the influences of it on the tunable features of bandgap. Recall that, the stable zone of NC includes two sets, namely, the left set $\alpha<-\left[\gamma\left(1-k_{31}^{2}\right)+1\right] /(\gamma+1)\left(1-k_{31}^{2}\right)$ and the right set $\alpha>-1$, as shown in Fig. 3. Accordingly, influences of $\mathrm{NC}$ will be analyzed separately in these two sets.

The cases with L and NC in series are first analyzed. Figure 5 (a), (b) and (c) show the variation of bandgap boundaries along with $\alpha$ in the left set of its stable zone for the covering ratios $\chi=0.5, \chi=0.7$ and $\chi=1$. The shadowed areas are the bandgap regions. The required $\mathrm{L}$ values for different $\alpha$ are also illustrated in these figures, the value is divided by $L_{0}$, which is the required inductance when no $\mathrm{NC}$ is connected with L. Figure 5 (d) illustrates the corresponding bandgap size variation along with $\alpha$. From Figs. 5 (a) and (b), it can be seen that for the situations with $\chi<1$, NC decreases simultaneously the upper and lower boundaries of the LR bandgap. The lower boundary drops faster than the upper one as $|\alpha|$ decreases, therefore, the bandgap size enlarges. As $\chi$ gets larger, the upper boundary becomes less sensitive to $\alpha$, and the bandgap is broadened more in these situations. The bandgap can be enlarged the most when $\chi=1$ since the upper boundary becomes immune to $\alpha$. From Figs. 5(a), (b) and (c), it is also 


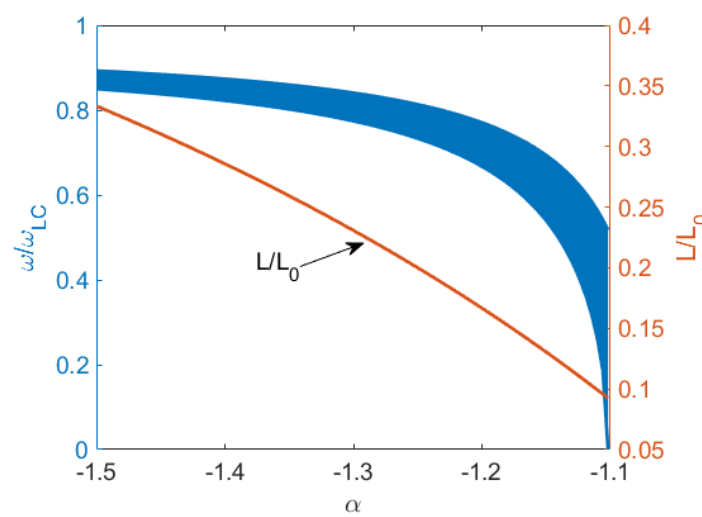

(a)

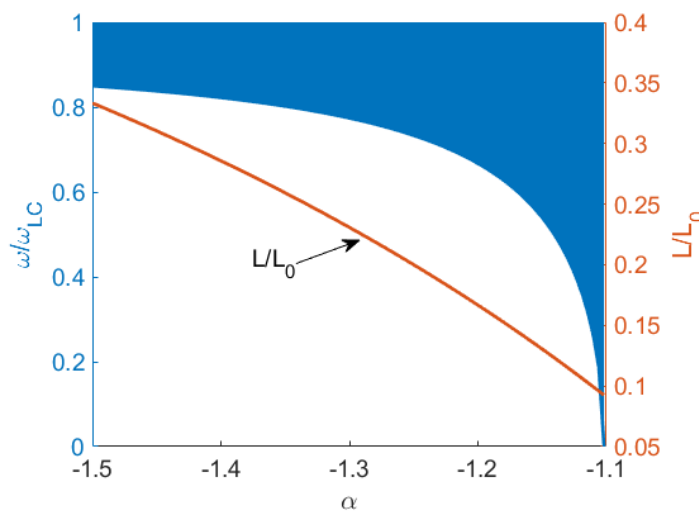

(c)

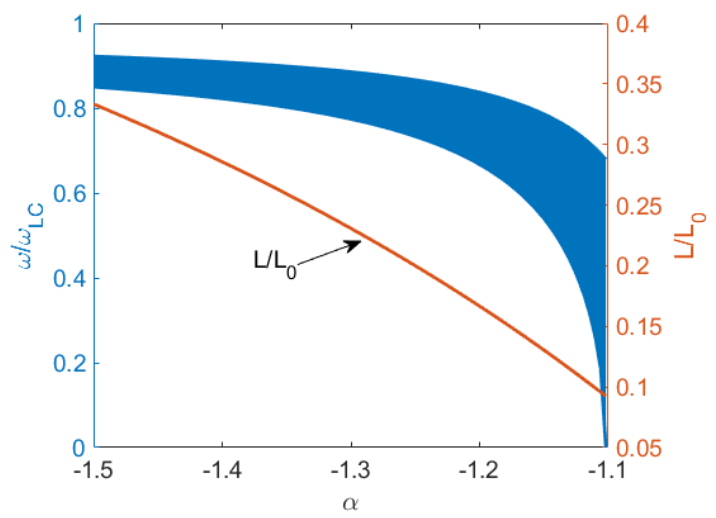

(b)

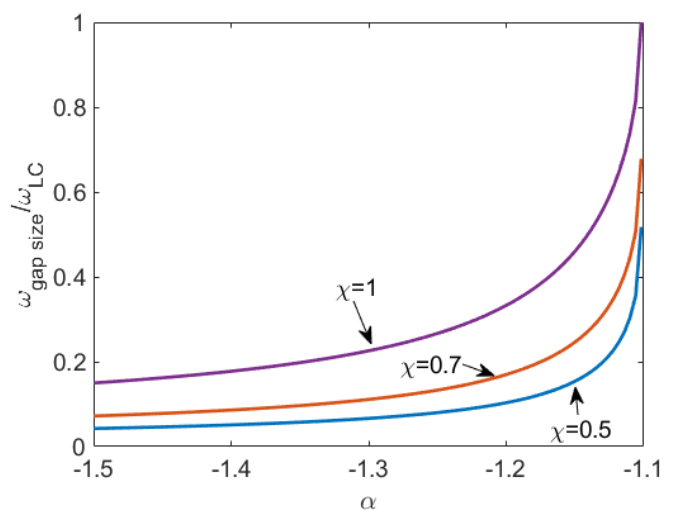

(d)

Figure 5: Variation of the bandgap boundaries (a-c) and size (d) along with $\alpha$ in the left set of its stable zone for the covering ratios (a) $\chi=0.5$, (b) $\chi=0.7$ and (c) $\chi=1$ when the $\mathrm{NC}$ is in series with the $\mathrm{L}$. The shadowed areas indicate the bandgap regions. The required $\mathrm{L}$ values for different $\alpha$ are also illustrated in Figs. 5(a), (b) and (c), $L_{0}$ is the required inductance when no $\mathrm{NC}$ is connected with $\mathrm{L}$.

seen that the required inductance value decreases as the bandgap enlarges. This makes the circuit become more easy to be realized in practice since large analog inductance is difficult to obtain. Note that if the shunts are realized using digital techniques, large inductance value is no longer a problem [24].

Figure 6 shows the variation of bandgap boundaries and size along with $\alpha$ in the right set of its stable zone. It can be observed that increasing the $|\alpha|$ results in increase of the upper and lower boundaries if $\chi<1$. Basically, the upper boundary gains fast than the lower one, leading to enlargement of the bandgap size. The lower boundary becomes less sensitive to $\alpha$ as $\chi$ gets larger. Consequently, the bandgap size can be improved more 


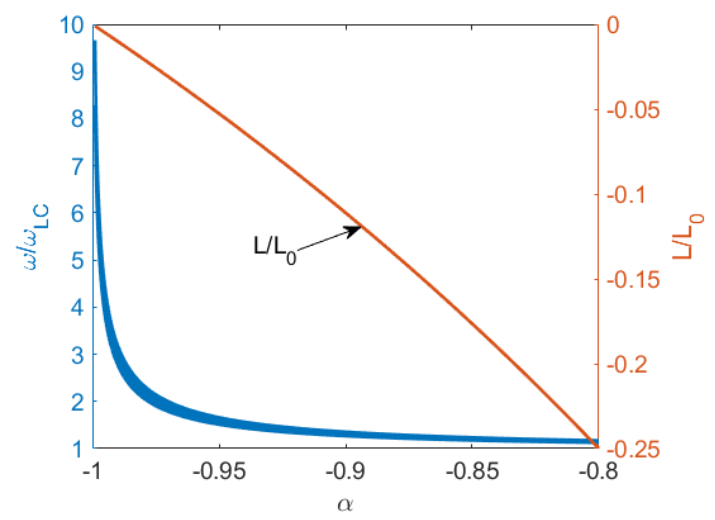

(a)

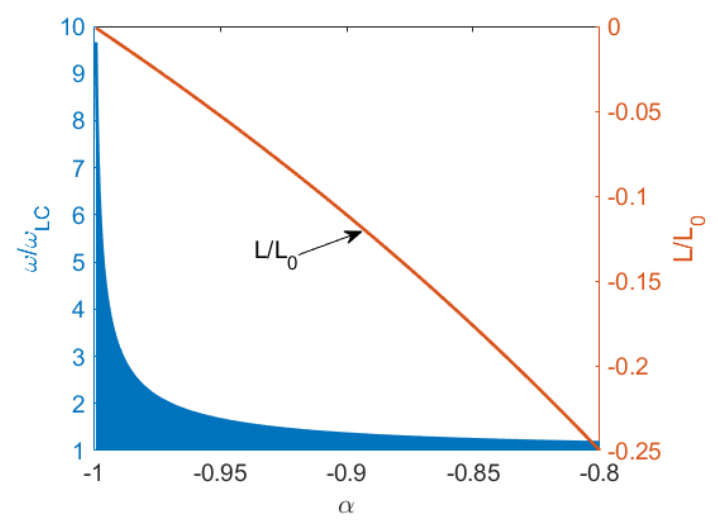

(c)

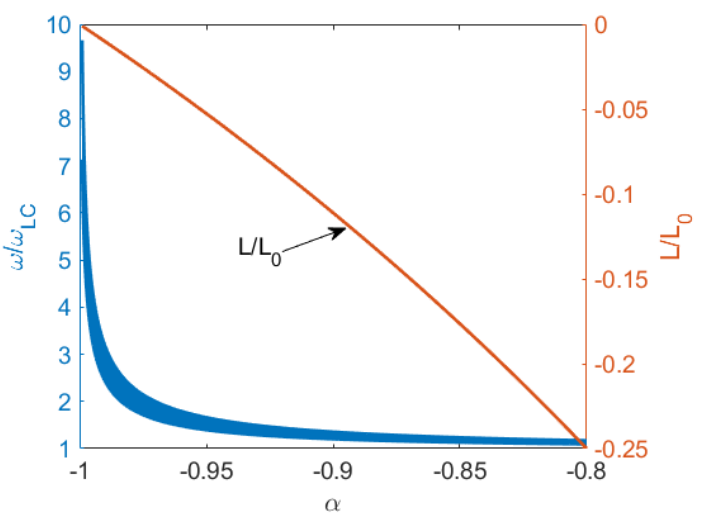

(b)

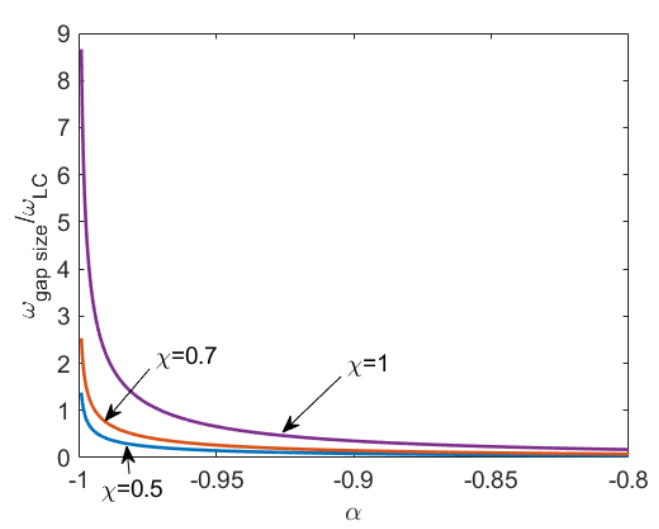

(d)

Figure 6: Variation of the bandgap boundaries (a-c) and size (d) and size along with $\alpha$ in the right set of its stable zone for the covering ratios (a) $\chi=0.5$, (b) $\chi=0.7$ and (c) $\chi=1$ when the NC is in series with the $\mathrm{L}$. The shadowed areas indicate the bandgap regions. The required $\mathrm{L}$ values for different $\alpha$ are also illustrated in Figs. 6(a), (b) and (c), $L_{0}$ is the required inductance when no NC is connected with $\mathrm{L}$.

for larger $\chi$. For the special situation with $\chi=1$, the upper boundary is left unchanged by NC, the largest bandgap can be achieved. The equivalent capacitance is negative in these circumstances, therefore, negative inductance values are also required according to $L=1 / \omega_{L C}^{2} C_{e q}$. It is also shown that inductance with a larger absolute value is needed to have wider bandgaps.

For the cases with L and $\mathrm{NC}$ in parallel, Figs 7 and 8 show the results corresponding to $\alpha$ inside the left set and the right set of its stable zone, respectively. Comparing to the case with $\mathrm{L}$ and $\mathrm{NC}$ in series, it is observed that in this case, the bandgap boundaries are oppositely increased within the left set and decreased within the right set. The trends 


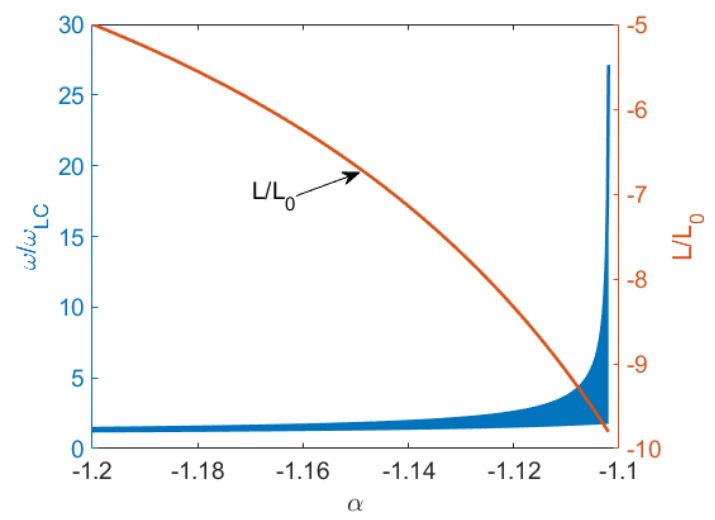

(a)

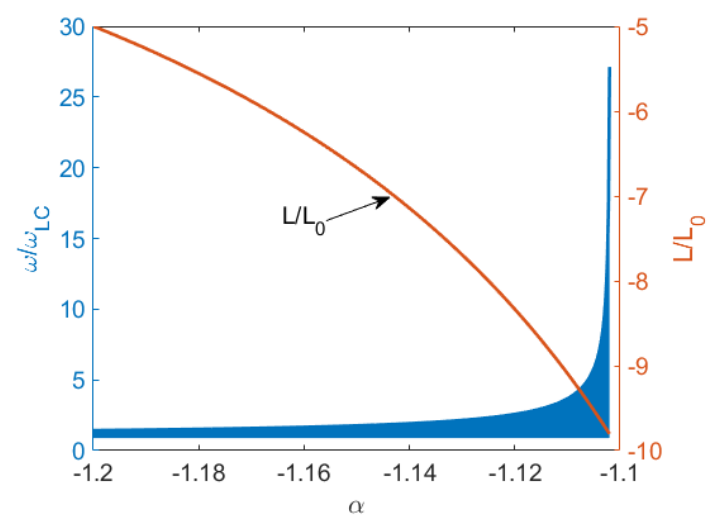

(c)

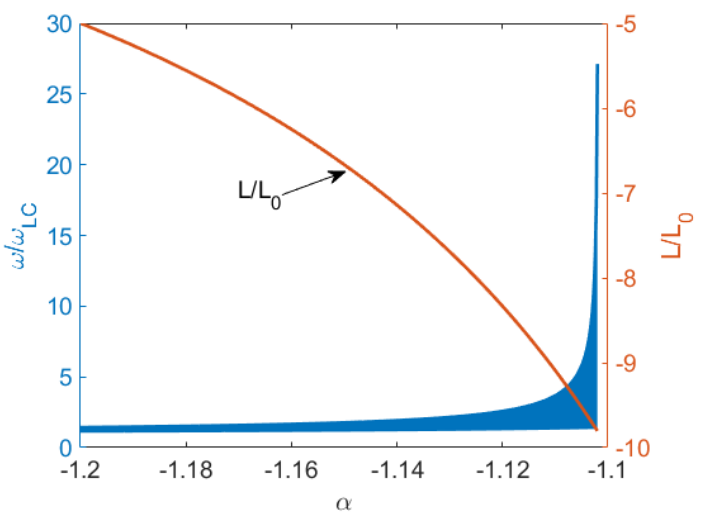

(b)

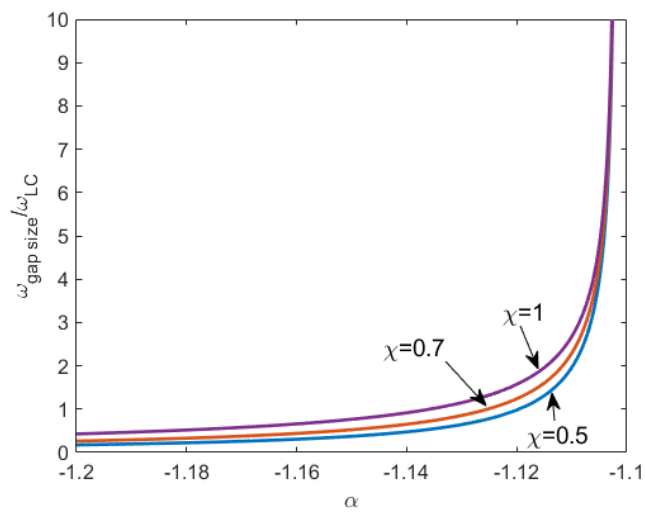

(d)

Figure 7: Variation of the bandgap boundaries (a-c) and size (d) and size along with $\alpha$ in the left set of its stable zone for the covering ratios (a) $\chi=0.5$, (b) $\chi=0.7$ and (c) $\chi=1$ when the $\mathrm{NC}$ is in parallel with the $\mathrm{L}$. The shadowed areas indicate the bandgap regions. The required $\mathrm{L}$ values for different $\alpha$ are also illustrated in Figs. 7(a), (b) and (c), $L_{0}$ is the required inductance when no NC is connected with $\mathrm{L}$.

of enlargement of bandgap size in Figs 7 and 6 are similar but not exactly the same, the obvious difference is that in Figs 7, the NC has little effect on the lower boundary of the gap even though the covering ratio is far smaller than 1. Comparing the results in Figs 8 and 5, it can be observed that the boundaries have similar variation pattern along with $\alpha$. However, it is noticed in Figs $8(\mathrm{~d})$ that, the gap size varies non-linearly with $\alpha$ when $\chi<1$, a maximum value appears before $\alpha$ reaches the stable zone limit.

Results in Figs. 5, 6, 7 and 8 give the conclusion that, to enlarge the LR bandgap, $\mathrm{NC}$ can either be in series or in parallel with $\mathrm{L}$, the $\mathrm{NC}$ value can be chosen from one of the two sets of its stable zone. Consequently, to design a bandgap covering a desired 


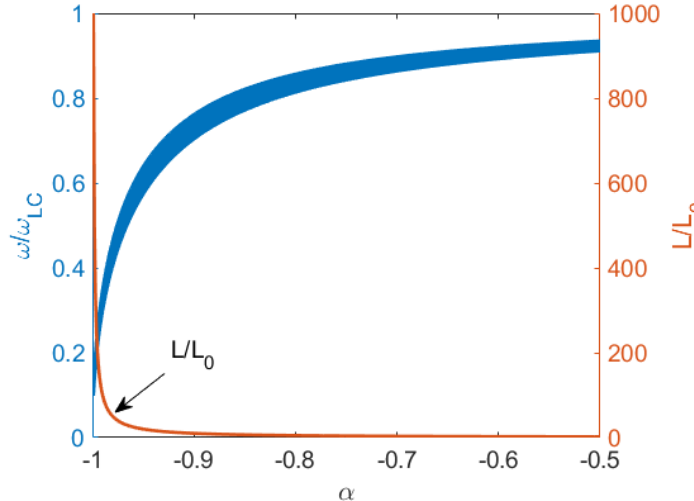

(a)

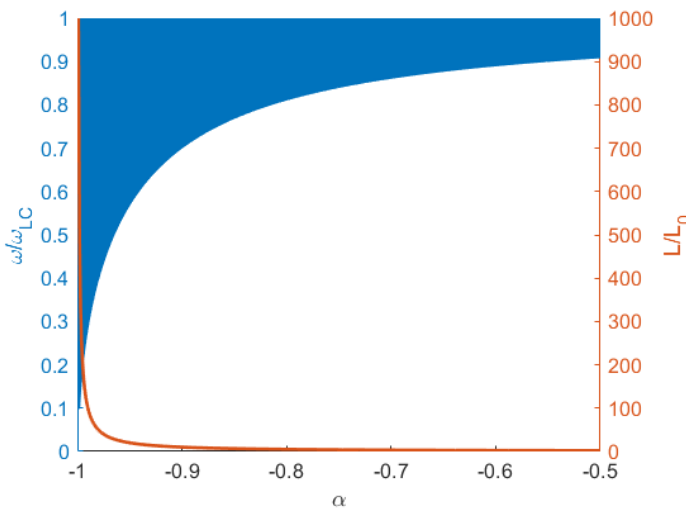

(c)

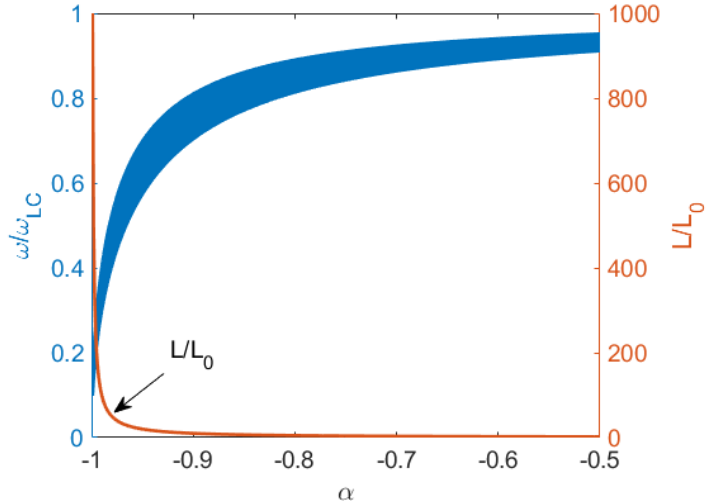

(b)

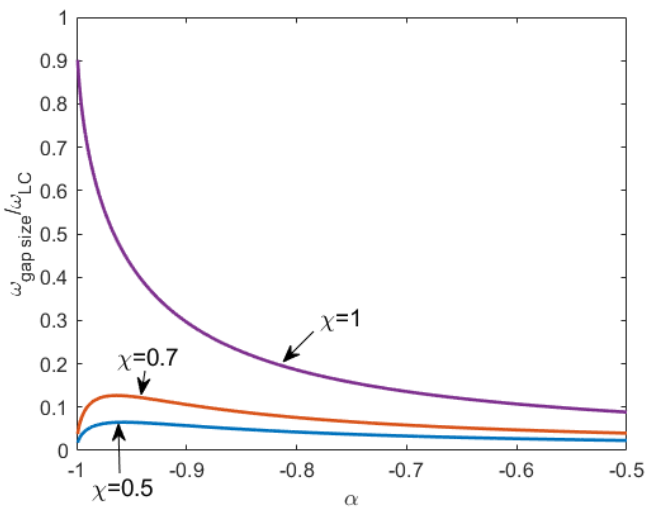

(d)

Figure 8: Variation of the bandgap boundaries (a-c) and size (d) and size along with $\alpha$ in the right set of its stable zone for the covering ratios (a) $\chi=0.5$, (b) $\chi=0.7$ and (c) $\chi=1$ when the $\mathrm{NC}$ is in parallel with the $\mathrm{L}$. The shadowed areas indicate the bandgap regions. The required $\mathrm{L}$ values for different $\alpha$ are also illustrated in Figs. 8(a), (b) and (c), $L_{0}$ is the required inductance when no NC is connected with L. 
frequency rang, one have 4 different choices in terms of shunt configurations and NC values. To avoid tedious discussions on similar results, in the following studies, only the cases using $\mathrm{NC}$ in series with $\mathrm{L}$ are considered, the $\mathrm{NC}$ values are limited within the left set of its stable zone.

The tuning characteristics of bandgap size by $\mathrm{NC}$ for different $\gamma$ and $k_{31}$ values are studied in Fig. 9. The right limit of the left set of the stable zone depends on $\gamma$ and $k_{31}$ (see Eq. (13)). Therefore, in Fig. 9 (a) and (c), $\alpha *(\gamma+1)\left(1-k_{31}^{2}\right) /\left[\gamma\left(1-k_{31}^{2}\right)+1\right]$ is used as the horizontal axis to eliminate the influences of the shifting of the stable zone's boundary. From Fig. 9 (a), it can be seen that the enlargement trend of the gap size caused by the varying of $\alpha$ is independent on $\gamma$. Considering $\gamma$ itself, from Fig. 9 (b) it is observed that the gap size varies non-linearly as $\gamma$ increases, the gap size enlarges in the beginning then decreases after a maximum. There is an optimal value for $\gamma$ in order to have the largest bandgap size, this value is independent on the NC value. The influences of $k_{31}$ are studied in Fig. 9 (c) and (d). $k_{31}$ varies when different piezoelectric material is used in the metamaterial. Therefore, knowing the effects of $k_{31}$ can help us in choosing the most suitable piezoelectric material in practice. Fig. 9 (c) shows the variation pattern of the gap size when $\alpha$ changes for different $k_{31}$ values. Absolute value of $k_{31}$ (its value is usually negative) is within 0.5 for typical piezoelectric ceramics in market. Accordingly, $\left|k_{31}\right|$ changes from 0.1 to 0.5 in Fig. 9 (c-d). It can be seen that $k_{31}$ also does not change the enlargement trend of the gap size when $\alpha$ varies. Besides, it is observed that for larger $\left|k_{31}\right|$, the gap size is less sensitive to the changing of $\alpha$ near the stable zone boundary, which makes the system more robust in practice.

Influences of $\mathrm{NC}$ on the imaginary part of wavenumber are studied in Fig. 10. To avoid singularity during the dispersion curve calculation, a small resistance $(R=50 \Omega)$ is introduced into the circuit in series with L and NC. The imaginary part of wavenumber is linked to the attenuation of waves within the bandgap. A larger absolute value of imaginary part means stronger wave attenuation effect. From Fig. 10 it is observed 


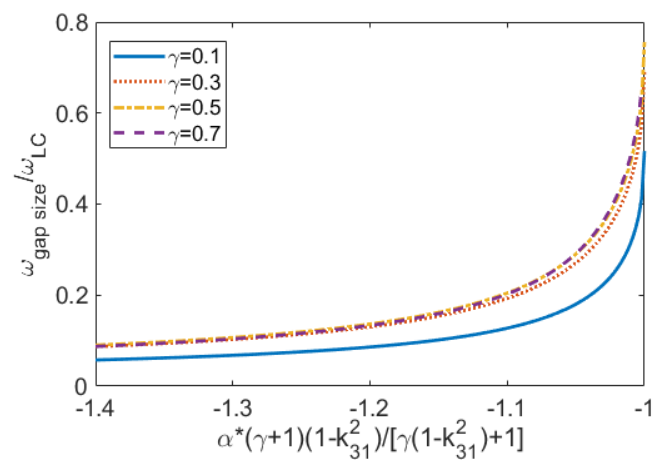

(a)

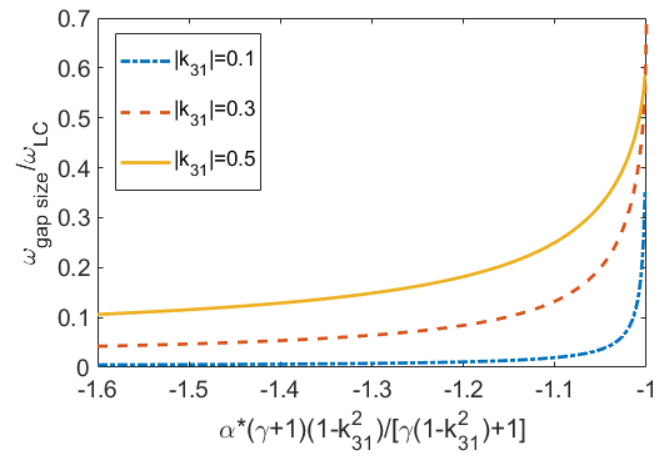

(c)

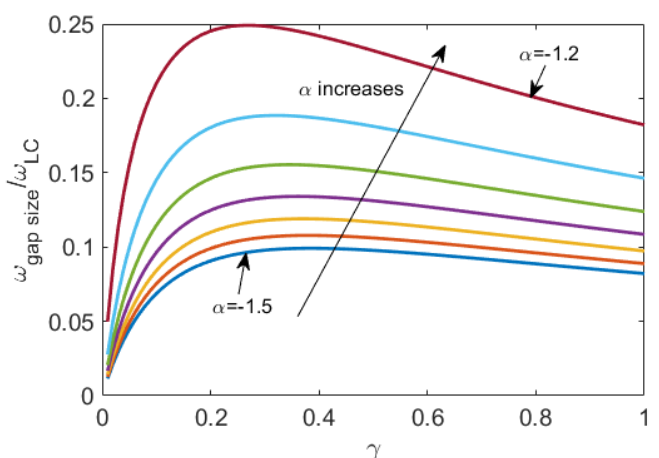

(b)

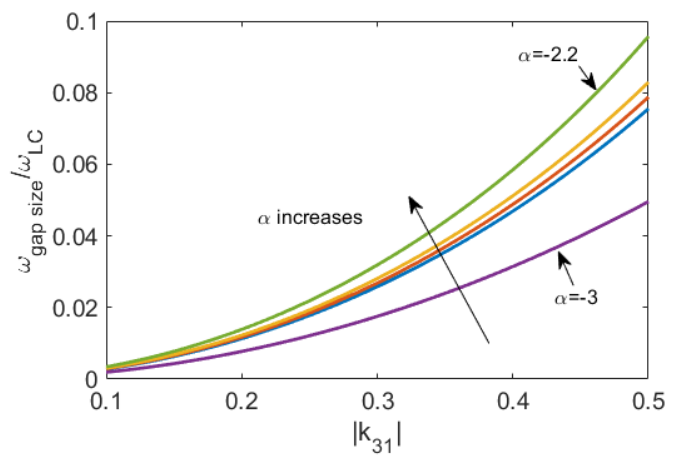

(d)

Figure 9: Influences of (a-b) $\gamma$ and (c-d) $k_{31}$ on the tuning characteristics of bandgap size by NC: (a) $\chi=0.8, k_{31}=-0.39$, (b) $\chi=0.8, k_{31}=-0.39$, (c) $\chi=0.8, \gamma=0.23$ and (d) $\chi=0.8, \gamma=0.23$. 


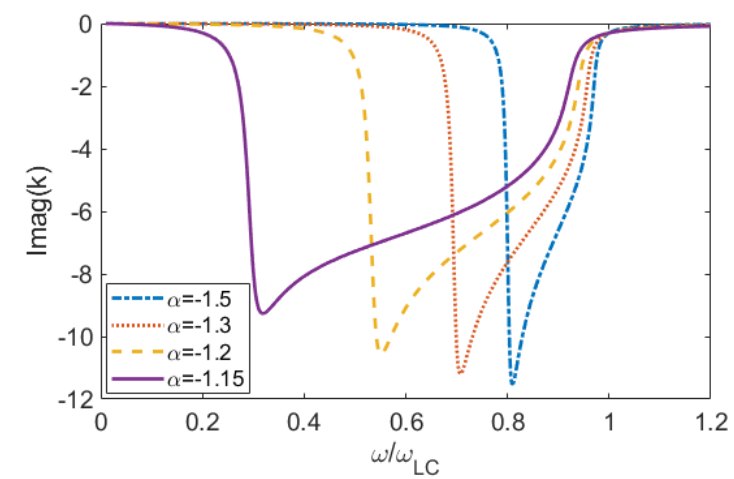

Figure 10: Influences of NC on the wave attenuation ability within the bandgap.

that NC broadens the bandgap size, but degenerates the wave attenuation ability. The degeneration is due to the enhancement of damping in the circuit by $\mathrm{NC}$, since damping weakens the resonant behavior of the circuit. Nonetheless, the degeneration is mild, the wave attenuation effects are strong within these bandgaps, as will be seen from the forced responses of the metamaterial beam in the following section.

In summary, studies in this section reveal how NC affects the bandgap when the piezoelectric metamaterial beam has different geometrical and material parameters. These results can be used as guidelines to design bandgaps at desired frequency ranges.

\section{Numerical simulations}

The main tuning characteristics of bandgap by NC are verified using finite element (FE) simulations. The simulations are done in commercial software COMSOL. The FE beam model has 10 unit cells, a harmonic boundary force is applied on the left end of the beam, the right end of the beam is free. Transmission function between the displacement of the right end and the excitation force will be studied during the discussions. Two different unit cell lengths are used, they are $l_{b}=52 \mathrm{~mm}$ and $l_{b}=100 \mathrm{~mm}$, the aim is to verify the influences of the covering ratio on the effects of NC. The other geometric and material parameters are the same as in Table 2. NC is in series with $\mathrm{L}$ in these simulations. In practice, resistance of the circuit is inevitable. Therefore, a small resistance $R=50 \Omega$ 
is also included in the shunt in series with the other parts.

First of all, accuracy of the FE models is checked and improved to be acceptable. The patches are kept OC to take into account the electro-mechanical coupling effects in these simulations. Natural frequencies of the first 10 non-rigid modes are used to check the convergence of the simulations. The meshes of FE models are refined until the simulated natural frequencies vary no more than $1 \%$ when the degrees of freedom are doubled.

Secondly, the intrinsic capacitance value of the patch in the FE models is estimated. This capacitance value is necessary in order to find the required inductance value when $\omega_{L C}$ is designated. In the analytical studies, $C_{p}^{s}$ is involved in the process to determine inductance values (for example, through $L=1 / \omega_{L C}^{2} C_{p}^{s}$ ). However, this analytical $C_{p}^{s}$ can not be directly used in the $\mathrm{FE}$ simulations to deign the $\mathrm{L}$ values, because it overestimates the capacitance of the patch in the FE model. To demonstrate this, the same $\mathrm{L}$ value is used in both the analytical and FE simulations, the analytically predicted bandgap range and the gap observed in the finite metamaterial beam are compared in Fig. 11 for the case with LR shunts. The analytical bandgap range (calculated using Eq. (12)) is indicated by the shadow in Fig. 11. The transmission function curve obtained in the FE simulation using the same parameters as those in the analytical calculation is labeled with "original", the frequency range with relatively low response level is the bandgap. It can be seen that the gap observed in the FE result is at higher frequency range than the analytical one, which verifies that the analytical $C_{p}^{s}$ overestimates the capacitance value of the patch in the FE model, leading to a smaller $\mathrm{L}$ than the required one. Therefore, the capacitance in the FE simulation is corrected to be $C_{p}^{s *}=0.84 C_{p}^{s}$. Using this corrected capacitance, the corrected L value for the FE simulation is obtained. The curve with label "corrected" in Fig. 11 is the FE result corresponding to the corrected $\mathrm{L}$ value. One can see that the gap in the FE simulation now has good coincidence with the analytical one.

After the above procedures, it is able to verify the effects of $\mathrm{NC}$ on the bandgaps. 4 shunts with different NC and L parameters are studied for each of the two unit cell 


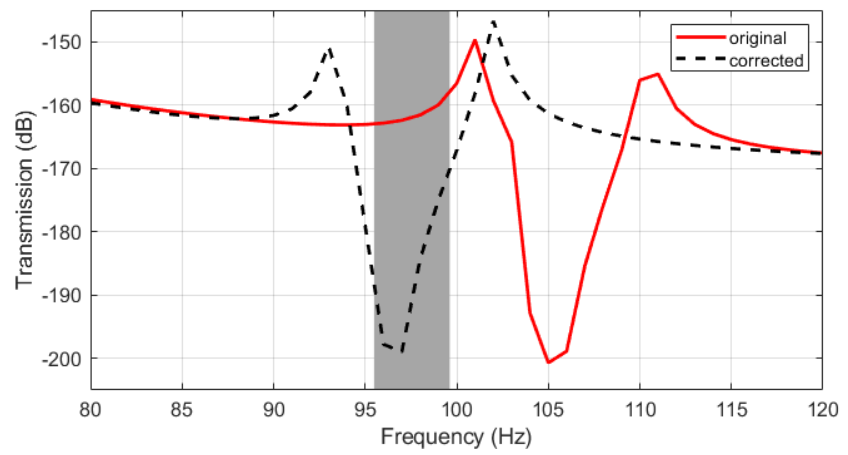

Figure 11: Transmissibility at the right end of the metamaterial beam when a boundary transverse force is applied at the left end for the case with LR shunts. Shadowed area indicates the analytically predicted gap region using Eq. (12), results corresponding to the original and corrected L are both shown in the figure.

lengths, the parameters of shunts are listed in Table 3 (shunt 1 to 4 ). The case with LR shunts is used as reference, the corresponding shunting parameters are also given in Table 3. Recall that, $C_{p}^{s *}$ in the table is the corrected capacitance of the patch in the FE model. $f_{L C}$ is the resonance frequency of the circuit in Hz. Figure 12 shows the transmission function curves for different studied cases. The predicted bandgap locations using Eqs. (12) and (14) are also indicated in these figures through the horizontal colorful slats. For the tuning trend of the bandgap size and location caused by $\mathrm{NC}$, it can be seen from the transmission curves in Fig. 12(a) that, when the covering ratio of the patch is close to one, NC decreases the lower boundaries of the gap, and has little influence on the upper ones, therefore, leading to a wider bandgap, just as illustrated in Figs. 5(c); when the covering ratio is far from one, the results in Fig. 12(b) clearly show that NC decreases simultaneously the upper and lower boundaries of the gap, the lower boundary drops faster, therefore, shifting as well as enlarging of the bandgap are both observed, which is coincident with the results in Figs. 5(a). From Fig. 12, it can also be seen that, with regard to the values of the bandgap sizes and locations, the FE and analytical results have obvious differences when there are NCs in the shunts, which indicates that the analytical model based on Euler-Bernoulli beam theory and sub-wavelength homogenization theory can not precisely capture the bandgap' features. 
Table 3: Resonance frequency of the circuit and the corresponding electrical parameters used in the FE simulations

\begin{tabular}{lllll}
\hline & $\mathrm{L}(\mathrm{H})$ & $\mathrm{R}(\Omega)$ & $C_{0} / C_{p}^{s *}$ & $f_{L C}(\mathrm{~Hz})$ \\
\hline reference & 12.00 & 50 & $\backslash$ & 100 \\
shunt 1 & 4.00 & 50 & -1.5 & 100 \\
shunt 2 & 2.40 & 50 & -1.25 & 100 \\
shunt 3 & 2.00 & 50 & -1.2 & 100 \\
shunt 4 & 1.57 & 50 & -1.15 & 100 \\
\hline
\end{tabular}

In conclusion of this section, the FE results clearly verify the tuning characteristics of the bandgap observed in the analytical studies, therefore, the analytical model could be used to predict the tuning trend of the bandgap, however, to precisely calculate the size and location of a bandgap, FE simulations are more suitable.

\section{Discussions}

To implement shunts with L and NC in practice, one can use analog circuits to synthesize negative capacitance and large inductance. In this way, it is recommended to use $\mathrm{L}$ in series with $\mathrm{NC}$ and choose the $\mathrm{NC}$ value from the left set of stable zone. This choice could avoid using simultaneously negative capacitance and negative inductance in the shunts, it also benefits from the smaller inductance needed for wider bandgaps (see Figs. 5). The required inductance and negative capacitance can be realized using the circuits in Fig. 13 (a) and (b), respectively [22, 29]. The synthesized inductance and negative capacitance are respectively

$$
L=\frac{R_{1} R_{3} R_{4}}{R_{2}} C, C_{0}=-\frac{R_{3}}{R_{2}} C .
$$

A more advanced way to realize shunts with $\mathrm{L}$ and $\mathrm{NC}$ is using programmable digital circuits [24]. A digital circuit usually is composed of voltage measuring block, digital signal processing (DSP) block and voltage controlled current source block. Digital circuit measures the voltage on the electrodes of PZT patches and feeds back current to the same patches, therefore desired impedance is established between the terminals of patches. 


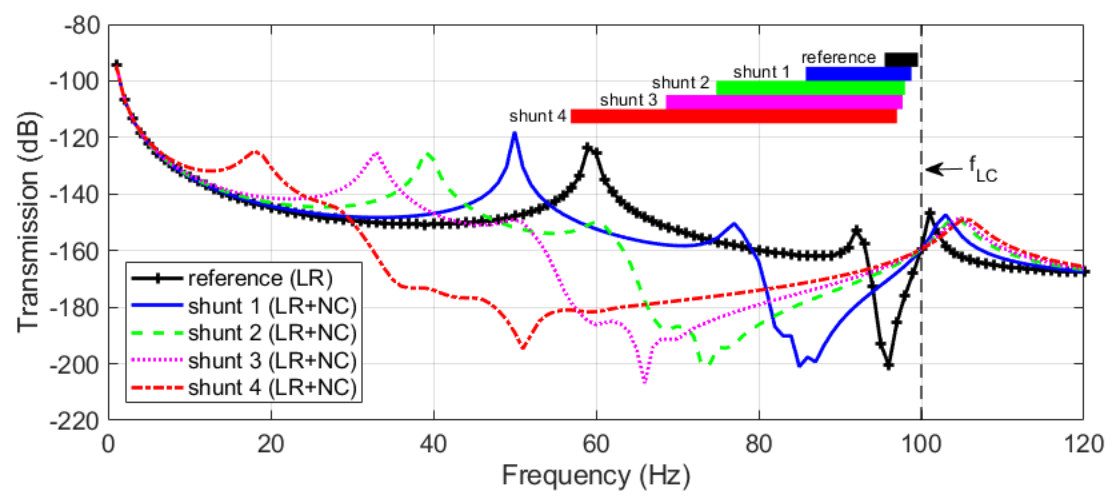

(a)

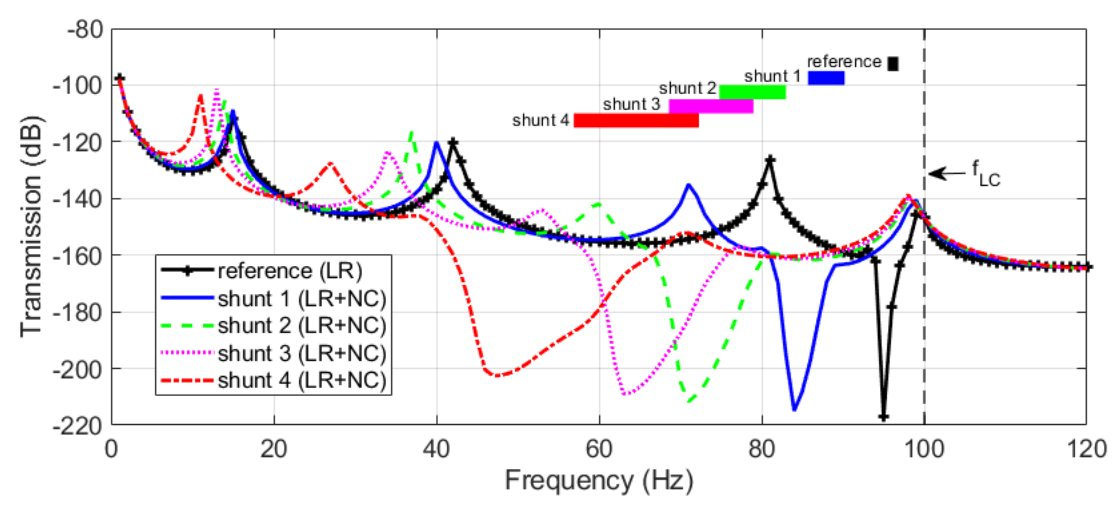

(b)

Figure 12: Transmission function curves for different patch covering ratios and shunts with different parameters: (a) $l_{b}=52 \mathrm{~mm}, l_{p}=50 \mathrm{~mm}$ and (b) $l_{b}=100 \mathrm{~mm}, l_{p}=50 \mathrm{~mm}$. The horizontal colorful slats in each figure indicate the predicted bandgap locations using Eqs. (12) and (14). The used shunting parameters in different simulations are listed in Table 3. 
(a)

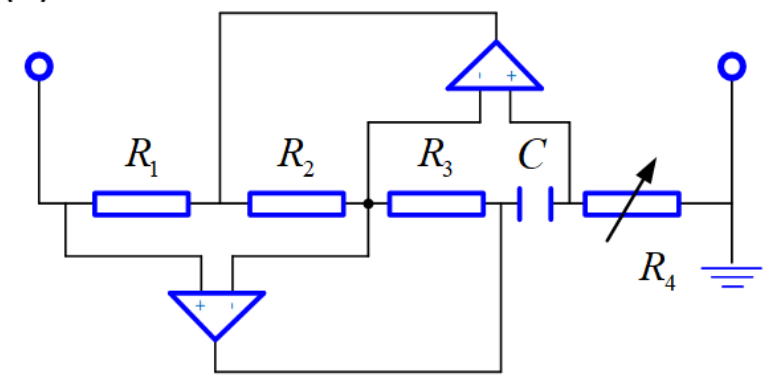

(b)

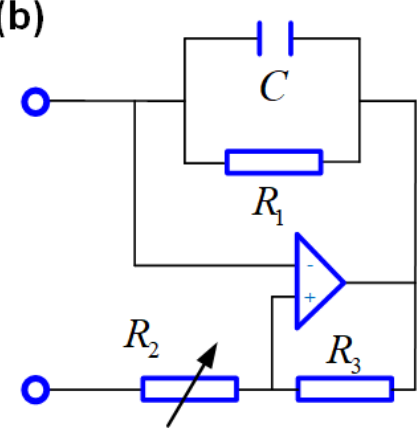

Figure 13: (a) Antoniou's circuit to synthesize inductance and (b) typical circuit to synthesize negative capacitance.

To simulate behaviors of shunts with L and NC, the inverse expression of the shunt's impedance is programmed in the DSP block in Laplace domain as a transfer function. Since the impedance is realized digitally, no limitation is constrained on the inductance value and the configurations of $\mathrm{L}$ with $\mathrm{NC}$, namely, the $\mathrm{L}$ and $\mathrm{NC}$ could either in series or in parallel, and it is free to choose $\mathrm{NC}$ value within the whole stable zone, as long as the implemented transfer function in the DSP block is causal and the whole system is stable.

The above discussed synthetic and digital circuits both need power supply in practice. One possible solution is introducing energy harvesting circuits into the system, the harvested energy maybe used to power low-powered electron devices in the circuits. Future work will be done to verify this idea.

\section{Conclusions}

In this paper, piezoelectric metamaterials shunted with combined $\mathrm{L}$ and $\mathrm{NC}$ are analytically and numerically studied. The metamaterial is obtained by periodically distributing piezoelectric patches on the surfaces of a host structure. A slender beam is used as host structure in this paper as an representative. Shunts with NC and L in series and in parallel are both studied. Major conclusions of this paper are:

(1) NC enlarges the frequency regions of negative stiffness caused by resonant shunts, therefore leading to wider bandgaps; 
(2) NC enlarges the LR bandgap size and also shifts the bandgap to lower or higher frequency range when the host beam is not fully covered by the patches. As the covering ratio of the patches increases, the gap size becomes even wider but the shifting effect of the bandgap location weakens;

(3) Except the covering ratio of patches, other geometric and material parameters of the metamaterial do not change the enlargement trend of the bandgap size caused by NC.

(4) The analytical model based on the Euler-Bernoulli beam theory and effective medium theory is useful to predict the tuning characteristics of bandgaps by NC. However, it can not precisely predict the sizes and locations of bandgaps, for such demand, FE models are more suitable.

In the next step, experiments will be done to study the influences of real NC and L circuits on the bandgaps and to reveal the limitations of such piezoelectric metamaterials under practical constrains.

\section{References}

[1] Z. Liu, X. Zhang, Y. Mao, Y. Y. Zhu, Z. Yang, C. T. Chan, and S. Ping. Locally resonant sonic materials. Science, 289(5485):1734-1736, 2000.

[2] R. Zhu, X. N. Liu, G. K. Hu, C. T. Sun, and G. L. Huang. A chiral elastic metamaterial beam for broadband vibration suppression. Journal of Sound and Vibration, 333(10):2759-2773, 2014.

[3] Jaesoon Jung, Hyun-Guk Kim, Seongyeol Goo, Kyoung-Jin Chang, and Semyung Wang. Realisation of a locally resonant metamaterial on the automobile panel structure to reduce noise radiation. Mechanical Systems and Signal Processing, 122:206$231,2019$.

[4] Christophe Droz, Olivier Robin, Mohamed Ichchou, and Noureddine Atalla. Improving sound transmission loss at ring frequency of a curved panel using tunable 
3D-printed small-scale resonators. The Journal of the Acoustical Society of America, 145(1):EL72-EL78, 2019.

[5] S. Timorian, M. Ouisse, N. Bouhaddi, S. De Rosa, and F. Franco. Numerical investigations and experimental measurements on the structural dynamic behaviour of quasi-periodic meta-materials. Mechanical Systems and Signal Processing, 136:106516, 2020.

[6] Changqi Cai, Jiaxi Zhou, Linchao Wu, Kai Wang, Daolin Xu, and Huajiang Ouyang. Design and numerical validation of quasi-zero-stiffness metamaterials for very lowfrequency band gaps. Composite Structures, 236:111862, 2020.

[7] M. Collet, KA K.a. A Cunefare, and MN N M.N. Ichchou. Wave Motion Optimization in Periodically Distributed Shunted Piezocomposite Beam Structures. Journal of Intelligent Material Systems and Structures, 20(7):787-808, 2008.

[8] M. Collet, M. Ouisse, and F. o. Adaptive metacomposites for vibroacoustic control applications. IEEE Sensors Journal, 14(7):2145-2152, 2014.

[9] Morvan Ouisse, Manuel Collet, and Fabrizio Scarpa. A piezo-shunted Kirigami auxetic lattice for adaptive elastic wave filtering. Smart Materials and Structures, In press, 25(11):1-12, 2016.

[10] Bin Bao, Daniel Guyomar, and Mickaël Lallart. Vibration reduction for smart periodic structures via periodic piezoelectric arrays with nonlinear interleaved-switched electronic networks. Mechanical Systems and Signal Processing, pages 1-29, 2016.

[11] Lin Li, Zhou Jiang, Yu Fan, and Jun Li. Creating the Coupled Band Gaps in Piezoelectric Composite Plates by Interconnected Electric Impedance. Materials, 11(9):1656, 2018. 
[12] Yan-Feng Wang, Yi-Ze Wang, Bin Wu, Weiqiu Chen, and Yue-Sheng Wang. Tunable and active phononic crystals and metamaterials. Applied Mechanics Reviews, 72(4), 2020 .

[13] N. W. Hagood and A. V. Flotow. Damping of structural vibrations with piezoelectric materials and passive electrical networks. Journal of Sound Vibration, 146(2):243268, 1991.

[14] O. Thorp, M. Ruzzene, and A. Baz. Attenuation and localization of wave propagation in rods with periodic shunted piezoelectric patches. In Spies International Symposium on Smart Structures Materials, 2001.

[15] L Airoldi and M Ruzzene. Design of tunable acoustic metamaterials through periodic arrays of resonant shunted piezos. New Journal of Physics, 13(11):113010, 2011.

[16] F Casadei, M Ruzzene, Lorenzo Dozio, and KA Cunefare. Broadband vibration control through periodic arrays of resonant shunts: experimental investigation on plates. Smart materials and structures, 19(1):015002, 2009.

[17] Filippo Casadei, Tommaso Delpero, Andrea Bergamini, Paolo Ermanni, and Massimo Ruzzene. Piezoelectric resonator arrays for tunable acoustic waveguides and metamaterials. Journal of Applied Physics, 112(6):064902, 2012.

[18] Shengbing Chen, Gang Wang, Jihong Wen, and Xisen Wen. Wave propagation and attenuation in plates with periodic arrays of shunted piezo-patches. Journal of Sound and Vibration, 332(6):1520-1532, 2013.

[19] C Sugino, M Ruzzene, and A Erturk. An analytical framework for locally resonant piezoelectric metamaterial plates. International Journal of Solids and Structures, $182: 281-294,2020$. 
[20] X. Xiao, Z. C. He, Eric Li, B. Zhou, and X. K. Li. A lightweight adaptive hybrid laminate metamaterial with higher design freedom for wave attenuation. Composite Structures, 243:112230, 2020.

[21] Gang Wang, Jianwei Wang, Shengbing Chen, and Jihong Wen. Vibration attenuations induced by periodic arrays of piezoelectric patches connected by enhanced resonant shunting circuits. Smart materials and structures, 20(12):125019, 2011.

[22] Gang Wang and Shengbing Chen. Large low-frequency vibration attenuation induced by arrays of piezoelectric patches shunted with amplifier-resonator feedback circuits. Smart Materials and Structures, 25(1):015004, 2015.

[23] Davide Cardella, Paolo Celli, and Stefano Gonella. Manipulating waves by distilling frequencies: a tunable shunt-enabled rainbow trap. Smart Materials and Structures, 25(8):085017, 2016.

[24] Kaijun Yi, Gaël Matten, Morvan Ouisse, Emeline Sadoulet-Reboul, Manuel Collet, and Gaël Chevallier. Programmable metamaterials with digital synthetic impedance circuits for vibration control. Smart Materials and Structures, 29(3):035005, 2020.

[25] L Airoldi and M Ruzzene. Wave propagation control in beams through periodic multibranch shunts. Journal of Intelligent Material Systems and Structures, 22(14):15671579, 2011.

[26] Gang Wang, Jianqing Cheng, Jingwei Chen, and Yunze He. Multi-resonant piezoelectric shunting induced by digital controllers for subwavelength elastic wave attenuation in smart metamaterial. Smart Materials and Structures, 26(2):025031, 2017.

[27] Christopher Sugino, Massimo Ruzzene, and Alper Erturk. Design and analysis of piezoelectric metamaterial beams with synthetic impedance shunt circuits. IEEE/ASME Transactions on Mechatronics, 23(5):2144-2155, 2018. 
[28] Manuel Collet, Morvan Ouisse, Mohamed Najib Ichchou, and Roger Ohayon. Semiactive optimization of $2 \mathrm{~d}$ wave dispersion into shunted piezo-composite systems for controlling acoustic interaction. Smart Materials and Structures, 21(9):094002, 2012.

[29] F Tateo, M Collet, M Ouisse, MN Ichchou, KA Cunefare, and P Abbe. Experimental characterization of a bi-dimensional array of negative capacitance piezo-patches for vibroacoustic control. Journal of Intelligent Material Systems and Structures, 26(8):952-964, 2015.

[30] Bin Bao and Quan Wang. Elastic wave manipulation in piezoelectric beam metastructure using electronic negative capacitance dual-adjacent/staggered connections. Composite Structures, 210:567-580, 2019.

[31] Manuel Collet, Ken A Cunefare, Massimo Ruzzene, and L Airoldi. Diffusion Draft : and Plates Energy Diffusion By Mean of Distributed Shunted Patches. In ASME 2009 Conference on Smart Materials, Adaptive Structures and Intelligent Systems, pages 211-216. American Society of Mechanical Engineers Digital Collection, 2009.

[32] Marta Berardengo, Olivier Thomas, Christophe Giraud-Audine, and Stefano Manzoni. Improved resistive shunt by means of negative capacitance: new circuit, performances and multi-mode control. Smart Materials and Structures, 25(7):075033, 2016.

[33] K. Yi, M. Collet, M. Ichchou, and L. Li. Flexural waves focusing through shunted piezoelectric patches. Smart Materials and Structures, 25(7):075007, 2016.

[34] Y. Fan, M. Collet, M. Ichchou, L. Li, O. Bareille, and Z. Dimitrijevic. A wave-based design of semi-active piezoelectric composites for broadband vibration control. Smart Materials and Structures, 25(5):055032, 2016.

[35] YY Chen, GL Huang, and CT Sun. Band gap control in an active elastic metamaterial 
with negative capacitance piezoelectric shunting. Journal of Vibration and Acoustics, 136(6), 2014.

[36] R. Zhu, Y.Y. Chen, M.V. Barnhart, G.K. Hu, C.T. Sun, and G.L. Huang. Experimental study of an adaptive elastic metamaterial controlled by electric circuits. Applied Physics Letters, 108(1):011905, 2016.

[37] YY Chen, GK Hu, and GL Huang. An adaptive metamaterial beam with hybrid shunting circuits for extremely broadband control of flexural waves. Smart Materials and Structures, 25(10):105036, 2016.

[38] Weijian Zhou, Weiqiu Chen, Zhenyu Chen, CW Lim, et al. Actively controllable flexural wave band gaps in beam-type acoustic metamaterials with shunted piezoelectric patches. European Journal of Mechanics-A/Solids, 77:103807, 2019.

[39] B. D. Marneffe. Active and passive vibration isolation and damping via shunted transducers. Thesis, Universite Libre De Bruxelles, 2007.

[40] M. Berardengo, S. Manzoni, O. Thomas, and M. Vanali. Piezoelectric resonant shunt enhancement by negative capacitances: Optimisation, performance and resonance cancellation. Journal of Intelligent Material Systems and Structures, 29(12):25812606, 2018.

[41] A. H. Meitzler, D. Berlincourt, F. S. Welsh, H. F. Tiersten, G. A. Coquin, and A. W. Warner. 176-1987 - ieee standard on piezoelectricity. 1988.

[42] Xiaoming Zhou and Gengkai Hu. Analytic model of elastic metamaterials with local resonances. Physical Review B, 79(19):195109, 2009.

[43] Hao Zhang, Jihong Wen, Yong Xiao, Gang Wang, and Xisen Wen. Sound transmission loss of metamaterial thin plates with periodic subwavelength arrays of shunted piezoelectric patches. Journal of Sound and Vibration, 343:104-120, 2015. 
[44] C. Sugino, S. Leadenham, M. Ruzzene, and A. Erturk. An investigation of electroelastic bandgap formation in locally resonant piezoelectric metastructures. Smart Materials Structures, 26(5):055029, 2017. 